\title{
Fill the Gap! Combining Pragmatic and Prosodic Information to Make Gapping Easy
}

\author{
John C. J. Hoeks • Gisela Redeker • Petra Hendriks
}

Published online: 5 April 2009

(C) The Author(s) 2009. This article is published with open access at Springerlink.com

\begin{abstract}
Two studies investigated the effects of prosody and pragmatic context on off-line and on-line processing of sentences like John greeted Paul yesterday and Ben today. Such sentences are ambiguous between the so-called 'nongapping' reading, where John greeted Ben, and the highly unpreferred 'gapping' reading, where Ben greeted Paul. In the first experiment, participants listened to dialogues and gave a speeded response as to which reading of an ambiguous target sentence first comes to mind. In the second experiment, they also responded to a visual probe that was presented during the presentation of the ambiguous target. The results show that context and prosody have independent and strong effects on both on-line processing and off-line interpretation of gapping; in the right combination they can make gapping as easy as the normally preferred nongapping reading.
\end{abstract}

Keywords Gapping $\cdot$ Pragmatic context $\cdot$ Prosody $\cdot$ Spoken language processing

\section{Introduction}

Ellipsis - the apparent deletion of linguistic material from an utterance without changing its truth-conditional meaning-is an ubiquitous phenomenon in language. In most cases it is associated with ease of processing; in fact, it is often rather infelicitous to explicitly use material that is normally absent. Some instances of 'gapping', however, seem to be an exception to this rule. Consider, for instance, sentence (1a).

J. C. J. Hoeks $(\bowtie) \cdot$ G. Redeker $\cdot$ P. Hendriks

Center for Language and Cognition Groningen, University of Groningen,

PO Box 716, 9700 AS Groningen, The Netherlands

e-mail: j.c.j.hoeks@rug.nl

J. C. J. Hoeks

BCN NeuroImaging Center, University of Groningen, PO Box 716,

9700 AS Groningen, The Netherlands 
1a. John greeted Paul yesterday and Ben today.

1b. John greeted Paul yesterday and Ben greeted Paul today.

1c. John greeted Paul yesterday and John greeted Ben today.

In this sentence it is impossible to uniquely identify which elements were left out; the sentence is ambiguous between reading (1b), where first John greeted Paul, and then Ben greeted Paul, and reading (1c), where John greeted both Paul and Ben. We will follow linguistic convention and call the first form of ellipsis, where verb and grammatical object are elided 'gapping' (1b) and the second one 'conjunction reduction', or 'nongapping' (1c).

A number of studies have shown that language users, when given the choice, have a strong preference for the nongapping interpretation. For instance, in a written questionnaire study, Carlson (2001) showed that in ambiguous sentences that were very similar to (1a), gapping interpretations are chosen only $4 \%$ of the time. Manipulation of the semantic parallelism between the entities in the sentence led to an increase in the number of gapping interpretations in sentences such as "John \{animate\} visited the office \{inanimate\} yesterday and Ben \{animate\} today", but only to $40 \%$. Carlson (2001) suggested that the major force resisting gapping is the well-known Minimal Attachment Principle: choose the simplest syntactic structure in terms of nodes of the respective syntactic trees (Frazier 1987). Because nongapping is syntactically less complex (i.e., its syntactic description requires fewer nodes), it should for that reason be the preferred option in case of ambiguity. Gapping only becomes the option of choice when thematic fit information is brought into play, as in "John baked cookies for his parents and Ben for his grandparents", where the nongapping interpretation (where John baked Ben for his grandparents) is practically excluded, due to its high implausibility. Here, $81 \%$ of instances received a gapping response (Carlson 2001).

\section{Gapping and Prosody}

In a follow-up experiment using auditory stimuli, Carlson (2001) manipulated the prosody of the ambiguous sentences, to either bias towards the gapping or towards the nongapping interpretation. Bias was accomplished by making use of the fact that placement of pitch accent strongly correlates with the presence of new or contrastive information (e.g., Lambrecht 1994). Let us take a closer look at sentence (1a). In both the gapping and the nongapping interpretation there are two pairs of elements in each conjunct that are contrasted. In the gapping reading (e.g., 1b), John and Ben make up the first pair of contrasted elements, and yesterday and today constitute the second pair, as in "JOHN greeted Paul YESTERDAY and $B E N$ greed Paut TODAY" (capitalization here indicates the presence of pitch accent). In the nongapping condition (e.g., 1c), however, the first pair of contrastive elements is different: it is formed by Paul and Ben as in "John greeted PAUL YESTERDAY and John greeted BEN TODAY". Here, John is clearly de-accented. Thus, gapping and nongapping readings of an ambiguous sentence have distinct prosodic realizations by which the listener can tell them apart. But does the listener use this information?

The answer is yes, according to Carlson's results. The listener does use these prosodic cues, but not to the extent that gapping can become the preferred interpretation; nongapping is always preferred, no matter how strong the prosodic bias might be. Gapping promoting prosody was shown to raise the percentage of gapping responses to $44 \%$, still leaving a majority of nongapping responses. Carlson concludes that prosody is indeed an important factor in the processing of gapping structures, but the very strong preference for nongapping structures is created by a structural factor, namely syntactic simplicity. 


\section{Gapping and Context}

Keller (2001) conducted off-line acceptability studies to investigate whether also contextual factors may be in part responsible for the unpreferredness of gapping, and whether providing the right context could indeed increase the acceptability of gapping sentences (cf. Kuno 1976). He found that gapping sentences that were made unambiguous by case marking (such as, e.g., "She accompanied the boy to school and he to university"), when presented in isolation, were significantly less acceptable than control sentences (such as, e.g., "She accompanied the boy to school and the girl to university", which, by the way, is still ambiguous). However, by using a specific context (e.g., "Where did Hanna and Michael accompany the boy to?") these unambiguous sentences were considered as acceptable as their nongapping counterparts. But why would context have this effect?

Question contexts such as those used by Keller set up a complex expectation regarding the information structure of the answer, that makes the gapping interpretation of the subsequent sentence acceptable and almost natural. First of all, the context for a large part determines what will be the most likely topic of the following sentence, where a topic can be roughly defined as the thing the utterance provides information about (which most of the time is the grammatical subject of a sentence). Hoeks et al. (2002) provide off-line and on-line evidence for their view that language users prefer to have one and only one topic in any given utterance (as is the case in nongapping), unless contextual or prosodic cues suggest otherwise. This preference for a single topic is predicted to lead to processing difficulty when a sentence has not one but two topics, as is, for instance, the case in sentence coordinations (e.g., "John [topic1] greeted Paul and Ben [topic2] laughed"). Presenting these sentences in a context promoting two topics (e.g., "What did John and Ben do?"), instead of the usual single topic, effectively eliminated this processing difficulty. This suggests that coordinated sentences are difficult not only because they are syntactically complex, but because they are complex in terms of topic structure. This is an important finding because gapping, much like sentence coordination, involves having two contrastive topics (e.g., "John [topic1] greeted Paul yesterday and Ben [topic2] greeted Paul today"). If the preceding context prepares the way for two topics, processing difficulty is predicted to decrease.

The second important feature of the context question is that it determines what will be the focus of the answer, with focus being the new information that is provided by the answer. In the gapping interpretation of the ambiguous sentence (1a), there are contrastive foci set up by the question word 'Where' in Keller's context sentences, suggesting to the reader or listener that the answer will contain (prepositional) phrases indicating locations: one location for John's action, and one location for Ben's action. And finally, the question context makes unambiguously clear that the fact that Paul was greeted is given information. This is important, because only information that is given, can be 'gapped', i.e., left out of the sentence (Kuno 1976).

\section{Speeded Auditory Decision: Can Gapping be Made Easy?}

As important as Keller's experiments may be in demonstrating the clear role of context in the processing of gapping, they do not answer the question of whether gapping can become the preferred structure in ambiguous structures: Recall that the sentences that were used in the Keller study were made unambiguous by means of case marking; it is unclear whether the language user will in the same way be affected by contextual factors when processing an ambiguous gapping sentence, where the nongapping reading is still the most preferred option. 
In addition, Keller presented his sentences in the written modality only, so the results do not say much about the workings of the factor prosody. In the experiments that are presented here, we wanted to find out under what circumstances gapping becomes the structure of choice by manipulating both context and prosody of ambiguous gapping sentences. Doing so will enable us to gauge the strength of each of these factors separately, but will also allow us to investigate how context and prosody interact during the processing of gapping structures.

We started out by conducting an experiment using a speeded auditory decision task, in which participants had to indicate as fast as possible what reading (i.e., gapping or nongapping) first came to mind after hearing the ambiguous target sentence. By stressing that there are no right or wrong answers, and by asking for a speeded response, we hope to be able to test which representation was actually active in the mind of the listener at the time of the dialogue: the more time (and the more task-irrelevant processing) between stimulus and response, the more likely it is that this memory trace will be disturbed. We used sentences such as (1a), which in Carlson's questionnaire study received a gapping reading only $4 \%$ of the time. If we can achieve a gapping percentage of over $50 \%$ in this specific set of sentences, then we may have identified the right prosodic and pragmatic means to make gapping easy.

\section{Experiment 1}

Method

\section{Participants}

Thirty-two native speakers of Dutch were paid for participating in experiments $1 \mathrm{a}$ and $1 \mathrm{~b}$ ( 27 female; mean age 21 years, age range 17-25). The imbalance in gender should not cause problems, because recent research has shown that, though there may be gender differences in the perception of emotional prosody, the sexes seem to be equally proficient when it comes to understanding linguistic prosody, which is of concern here (Raithel and Hielscher-Fastabend 2004).

\section{Materials}

Thirty-two sets of mini-dialogues were constructed, consisting of a context, a target sentence, and a verification statement. Each set consisted of four versions of a given dialogue, obtained by crossing the factor Context (consistent with gapping vs. consistent with nongapping) with the factor Prosody (consistent with gapping vs. consistent with nongapping). Examples are shown in $2 \mathrm{a}-\mathrm{d}$. Note that English translations are given of the original Dutch stimuli (target sentences are structurally identical between languages); CAPITALS indicate the presence of a pitch accent.

\section{2a. (GG) Gapping Context, Gapping Prosody}

Context: Wilma has bought a new house. What did Nathan and Tessa help her with?

Target: NATHAN helped Wilma with PAINTING and TESSA with WALLPAPERING. Verification Statement (Gapping): Tessa helped Wilma.

\section{2b. (GN) Gapping Context, Nongapping Prosody}

Context: Wilma has bought a new house. What did Nathan and Tessa help her with? Target: Nathan helped WILMA with PAINTING and TESSA with WALLPAPERING. Verification Statement (Gapping): Tessa helped Wilma. 


\section{2c. (NG) Nongapping Context, Gapping Prosody}

Context: Wilma and Tessa have each bought a new house. What did Nathan help them with?

Target: NATHAN helped Wilma with PAINTING and TESSA with WALLPAPERING. Verification Statement (Gapping): Tessa helped Wilma.

\section{2d. (NN) Nongapping Context, Nongapping Prosody}

Context: Wilma and Tessa have each bought a new house. What did Nathan help them with?

Target: Nathan helped WILMA with PAINTING and TESSA with WALLPAPERING. Verification Statement (Gapping): Tessa helped Wilma.

Contexts consisted of a declarative sentence followed by a direct question, as shown in the context part of $2 \mathrm{a}-\mathrm{d}$. As already mentioned in our discussion of Keller (2001), this kind of context can serve three purposes: 1) suggesting the most likely topics for the following ambiguous elliptical sentence (i.e., two topics when biasing towards the gapping interpretation, one topic when biasing towards the nongapping interpretation); 2) suggesting the most likely focus for the subsequent sentence by asking for a time, a location, an instrument, etc.; 3) providing background information on the nature of the event ('to help'), and on the Patient associated with the event ('Wilma'), so that this combined information ('Wilma was helped') can be considered 'given' information that can be elided in the gapping reading of the ambiguous sentence.

As for the factor prosody, we know that prosody is a multi-dimensional construct, and it would be very interesting to see what aspects of it (e.g., amplitude variations, duration of pauses etc.) play a role in the processing of gapping structures. However, for our present experiments we will focus on the role of pitch contours, which have been shown to crucially contribute to sentence comprehension (Cutler et al. 1997). Figure 1 shows the pitch contours of the example sentence in both prosodic realizations. What distinguishes the two prosodies is the pattern of pitch accents in the first conjunct. In the prosodic pattern promoting gapping (see Fig. 1, upper panel), the name of the direct object, Wilma, is de-accented because it is background knowledge, which is required if this information is to be left out of the second conjunct. The name of the subject, Nathan, does receive an accent, because it is part of the contrastive topic construction involving both Nathan and Tessa. Each of them is expected to perform some action. In the nongapping condition (see Fig. 1, lower panel), the pattern of pitch accents in the first conjunct is completely reversed. The name of the grammatical subject, Nathan, is now de-accented, as it is the single topic of the sentence, and thus does not receive pitch accent (e.g., Lambrecht 1994). In this case there are no contrastive topics. What is contrasted here is the direct object in the first conjunct, Wilma, which is set against the purported grammatical object in the second conjunct, Tessa. Because of this contrastive relation, both receive pitch accent.

Summarizing, we have two factors, Context and Prosody, each of which is either consistent with gapping or consistent with nongapping. The experimental materials thus appear in four conditions created by crossing these two factors. It is important to note that two of the combinations (i.e., $2 \mathrm{~b}$ and $2 \mathrm{c}$ ) are infelicitous, because they contain a mismatch between the expectations set up by the context and the actual prosodic realization in the target sentence. Comparing the results of these two specific conditions will show which one of the two factors is the most influential with respect to interpretation: the linguistic context or the prosody of the target sentence. 

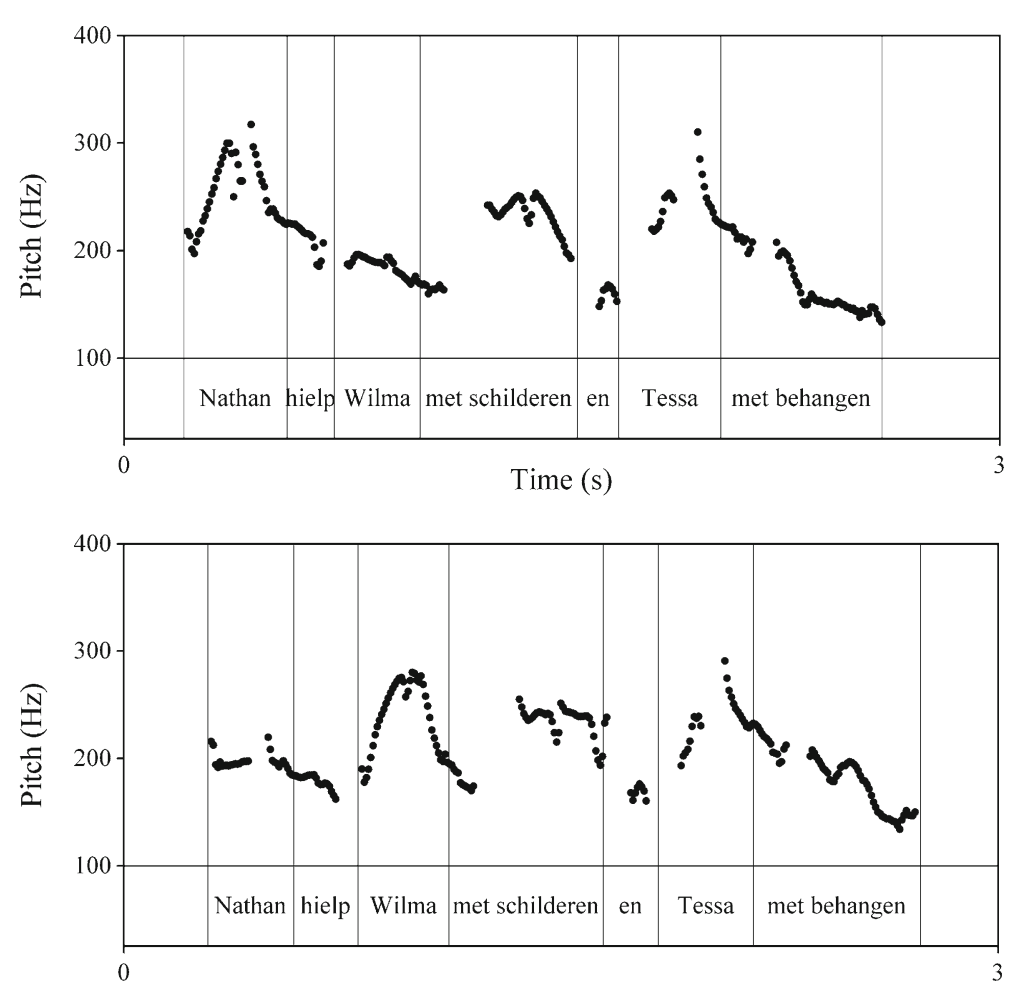

Time (s)

Fig. 1 Pitch contours of an example target sentence. Upper panel: prosody promoting gapping; Lower panel: prosody promoting nongapping

\section{Design}

Four experimental lists were constructed with 8 experimental dialogues per condition, and no list containing more than one version of a given item. Added to these 32 dialogues were another 32 dialogues from a related experiment, which served as fillers for the present experiment (for details, see Hoeks et al. 2006). The order in which experimental and filler items appeared was determined semi-randomly and was the same for each list. Each list was presented to an equal number of participants and each participant saw only one list. All verification statements represented the gapping reading of the ambiguous target sentence. The verification statements belonging to the filler items, however, all conformed to the nongapping reading of the ambiguous target sentence. Thus, participants were presented with an equal number of gapping and nongapping sentences (i.e., 32 of each). A practice session consisting of 16 dialogues preceded the actual experiment.

\section{Procedure}

Participants listened to the dialogues which were presented via two loudspeakers, while sitting behind a computer screen in a sound-proof booth. Each dialogue was preceded by a range of three asterisks appearing in the center of the computer screen (“***”), warning the 
participants that a new item would start. After $1060 \mathrm{~ms}$, the context sentence, spoken by a male speaker, was played, followed by the target sentence, spoken by a female speaker. The verification statement (male speaker again) was played subsequently, together with a visual presentation of three question marks (“???”), indicating that the participants should make a response.

Participants were specifically instructed to indicate whether the verification statement corresponded with the statement made by the female speaker (i.e., the target sentence). They could use the right SHIFT key on a keyboard for "YES" and the left SHIFT key for "NO". Participants were encouraged to respond as fast as they could and to follow their first impression; it was stressed that there were no 'correct' or 'incorrect' answers.

Results

Analysis

Response times were calculated separately for "YES" and "NO" responses. A "YES" response is always a choice for the gapping reading, as all verification statements were consistent with gapping; "NO" responses are thus considered choices for nongapping. The proportion of "YES" responses was also determined for each participant and each item. On all dependent measures, two analyses were performed: an $F$ 1-ANOVA on participant means for each condition and an F2-ANOVA on item means. The factors Context (promoting gapping vs. promoting nongapping) and Prosody (promoting gapping vs. promoting nongapping) were treated as within-participants and within-items factors. In addition, a planned comparison was performed between the two conditions where context and prosody offered conflicting evidence (see $2 b$ and $2 c$ ). Mean response times (in ms) and mean proportions gapping response are presented graphically in Fig. 2.

\section{Proportion Gapping Responses}

The main effects of Context and of Prosody were highly significant on both participantand item-analyses (all $p$-values $<.0001$ ). These results were qualified, however, by a significant two-way interaction between Context and Prosody $(F 1(1,31)=8.20, p<.01$; $F 2(1,31)=8.62, p<.01)$. This interaction suggested that the effect of prosody favoring gapping was stronger when the context also favored gapping (i.e., an effect of $25 \%=$ difference between "GG" and "GN"), than when the context did not (i.e., an effect of $8 \%=$ difference between "NG" and "NN"). Post-hoc tests revealed that both these effects were significant $(p<.05)$. The planned comparison showed that the condition where the context biased towards gapping responses and the prosody did not (i.e., "GN") produced significantly more gapping responses $(37 \% ; \mathrm{SE}=5)$ than in the condition where the context did not favor gapping, but the prosody did (i.e., "NG"; $17 \%$; $\mathrm{SE}=4$ ). The highest proportion of gapping responses, $61 \%$, was found in the "GG" condition, where both context and prosody promoted gapping.

\section{Response Times “YES” Responses}

Because in some of the conditions "YES" responses are unlikely or even absent (with fewest "YES" responses in the "NN" condition, see upper panel of Fig. 2), there was a considerable number of empty cells in the Repeated Measures ANOVA. The number of participants upon which to base this analysis became so small (and with that, the statistical power of the test) that 
Fig. 2 Upper panel: Proportion

"Yes" to gapping. Middle panel:

Decision times "Yes" to gapping.

Lower panel: Decision times

"No" to gapping.

"GG" = Gapping context,

Gapping prosody;

"GN"= Gapping context,

Nongapping prosody;

"NG" = Nongapping context,

Gapping prosody;

"NN" = Nongapping context,

Nongapping prosody. Error bars represent $1 \mathrm{SE}$
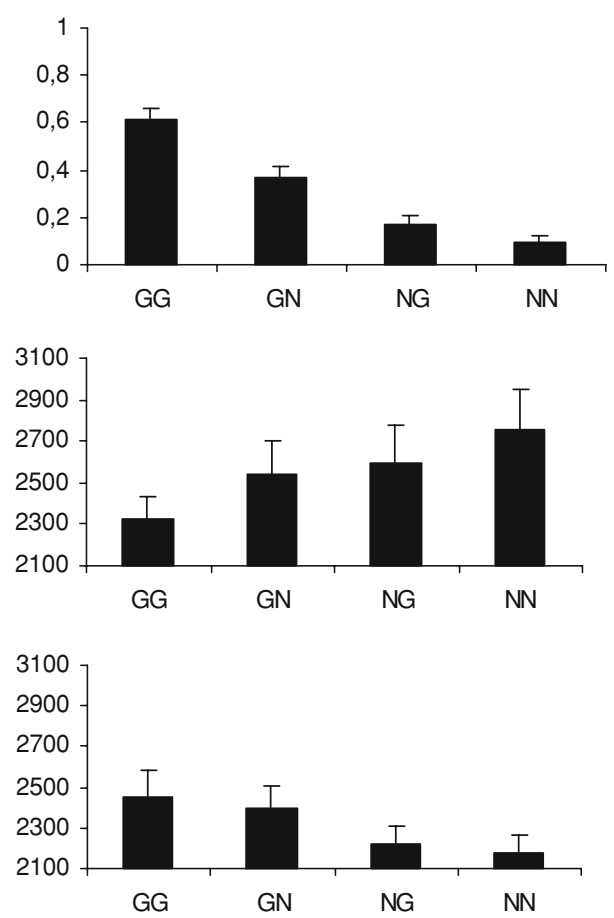

we decided to perform a Univariate ANOVA (which can be seen as stricter than the repeated measures ANOVA, as it tests against a larger error variance), with Context and Prosody as fixed between-group factors. The main effect of Context was significant in the analysis by items, though not by participants $(F 1(1,83)=3.34, p=.13 ; F 2(1,98)=10.18, p<.01)$. This main effect reflected the shorter response times for accepting the gapping interpretation of the target sentence when the context promoted gapping ( $2431 \mathrm{~ms}$; SE $=96$ ) than when it $\operatorname{did} \operatorname{not}(2677 \mathrm{~ms} ; \mathrm{SE}=128)$. No main effect of Prosody was found, nor was there a significant interaction (all $p$-values $>.20$ ).

\section{Response Times “NO” Responses}

For ease of comparison with the previous section we will report the results for the Univariate ANOVA (the outcome of the Repeated Measures ANOVA that we also ran was practically identical). The main effect of Context was significant by participants and by items $(F 1(1,120)=5.10, p<.05 ; F 2(1,124)=25.63, p<.001)$. Thus, when the context favored gapping, it took participants significantly longer to reject the gapping interpretation $(2427 \mathrm{~ms} ; \mathrm{SE}=73)$ than when the context did not $(2199 \mathrm{~ms} ; \mathrm{SE}=70)$. No main effect of Prosody was found, nor was there a significant interaction (all $F$-values $<1$ ).

\section{Discussion Experiment 1}

We wanted to find out whether it was possible to make the gapping reading the preferred interpretation of an ambiguous structure containing ellipsis. If we could, we should see a 
much higher percentage than the $4 \%$ gapping responses these sentences receive in isolation (see Carlson 2001). And indeed, we found that given the right context and the right prosody, participants chose gapping over $60 \%$ of the time (which is significantly different from chance expectation $(=50 \%) ; p<.05)$. Importantly, the pattern of reaction times convincingly shows that there is no sign of any speed-accuracy trade-off; on the contrary, there seems to be a strong linear relationship between proportion of gapping responses given, the ease and speed with which they are given, and the difficulty of rejecting the gapping response. These results suggest that, even if structural considerations were to play a role in resolving ellipsis, they can be overcome by the right combination of pragmatic and prosodic information.

In addition, we wanted to know which one of the two factors, context or prosody, was the most important for choosing gapping. Our results are very clear in showing that prosody and context both have an independent contribution to the processing of gapping structures. For instance, the effect of prosody in promoting gapping responses is significant in both the gapping and the nongapping context. However, our results also indicate that in the event of context and prosody pointing to different directions, context wins. That is, there are significantly more gapping responses when context favors gapping and prosody does not ("GN"), than when it is prosody that favors gapping, and the context does not ("NG"). Furthermore, the analyses on response times were very clear in that they showed only a significant main effect of Context, but not of Prosody. Thus, linguistic context outweighs prosody in the processing of sentences with ellipsis; in combination these factors are strong enough to overrule the strong preference for nongapping.

However, despite the evidence for the strong (combined) effects of context and prosody, and despite the fact that it was possible to make gapping the structure of choice in ambiguous sentences, these results were obtained via an off-line task. This makes it difficult to draw hard and fast conclusions about what is going on during the actual on-line processing of the ambiguous sentence. In other words, because the task measured off-line interpretation it does not permit us to choose between two options: 1) the on-line preference for nongapping did not go away, and did lead to processing difficulty during on-line processing, but due to the available contextual and prosodic information, reanalysis was completed before the end of the sentence, and 2) the preference for nongapping was overruled by context and prosody from the start, so there was no processing difficulty whatsoever. To address this issue, we conducted the exact same experiment as we described above, but this time we added an extra task to probe on-line processing: cross-modal gender decision.

\section{Cross-Modal Gender Decision: Is Gapping Really That Easy?}

In Experiment 2, we used the speeded auditory decision task that was used in the previous experiment. However, to find out whether there are differences in on-line processing between gapping and nongapping interpretations, two kinds of visual probes, in both cases proper nouns, were presented near the end of the sentence to estimate the ease of processing associated with gapping, and participants had to decide both quickly and accurately whether a probe was a girl's name or a boy's name, hence the term 'gender decision'. How does it work?

A sentence such as "Nathan helped Wilma with painting and Tessa with wallpapering" is globally ambiguous. There are no contextual or semantic cues as to which is the correct interpretation of the sentence. A strong linguistic context of the sort we are using in our experiments (e.g., "What did Nathan and Tessa help her with?") might make 
listeners reluctant to choose the nongapping reading (where only Nathan is doing the helping). However, it does not make gapping necessary, at least not from the start: Up to and including the third proper noun (Tessa), it is possible to continue the sentence as a standard sentence coordination (e.g., "Nathan helped Wilma with painting and Tessa vacuumed the living room").

Only at the second preposition (i.e., the 'with' of 'with wallpapering') is it unambiguously clear that we are dealing with gapping ("Nathan helped Wilma with painting and Tessa helped Wilma with wallpapering"). If gapping is resolved immediately, this would mean that from that point onward the elided material ('helped Wilma') is presumably reactivated in some form or other. Indeed, Kaan et al. (2004) have found evidence for the immediate reconstruction of the elided verb at the earliest possible point in gapping sentences. This will have the effect that the concepts denoted by 'helped' and by 'Wilma' are strongly activated at that point in the utterance. In contrast, when listeners go for the nongapping interpretation, "Nathan helped Wilma with painting and Nathan helped Tessa with wallpapering", there is no reason to assume that the concept associated with 'Wilma' is activated at the second preposition, at least not to the same degree as in the gapping interpretation.

To find out whether or not the concept 'Wilma' is activated, we presented a visual probe at the starting point of the second preposition. In one condition this probe was identical to the proper noun contained in the gapped constituent, in this example the name 'Wilma'. So with respect to this identity probe, we expect faster recognition in conditions with a predominant gapping response as compared to conditions where nongapping is the prepotent response. The other type of probe, the control probe, is constructed to bear superficial phonological resemblance with the identity probe (supposedly making the task more difficult for reasons stated below), but is different from any of the names used in the experiment. As the recognition of the control probe does not primarily depend on the level of activation of the concept belonging to 'Wilma', we assume that recognizing this control probe is mainly sensitive to the amount of processing difficulty occurring in the sentence where the probe is placed. In other words, control probes mainly measure processing load.

To try and avoid floor effects for recognizing the identity probes, all probes were visually 'degraded' by using a different font size and font style for every character of the name (also mixing capitals and normal letters). Furthermore, we reduced visual contrast by presenting the probes in lightgray on a white screen. The rationale is that by making the process of recognition longer and more difficult, effects of priming should become more visible (Holcomb 1993). The same reasoning underlies our choice for control probes that are to some extent phonologically similar to the identity probes: having partially similar probes should encourage our participants to make a decision only when the probe is fully identified, and not to give a reaction based on partial identification. To avoid memory related strategies we did not ask participants to say whether or not they think the probe occurred in the utterance, but merely to decide whether the name was typically a boy's or a girl's name.

\section{Experiment 2}

Method

Participants

Thirty-six native speakers of Dutch were paid for participating in this experiment ( 24 female; mean age 21 years, age range 19-27). 


\section{Materials \& Design}

For this experiment, 48 sets of mini-dialogues were used (including the 32 dialogues from Experiment 1). In the previous experiment, there were four conditions, obtained by crossing Context (consistent with gapping vs. consistent with nongapping) with Prosody (consistent with gapping vs. consistent with nongapping). For entirely practical reasons (i.e., to have at least eight items per condition), we chose to use only three of these four conditions, which combined with the factor Probe Type (identical vs. control) resulted in 6 new conditions. Six lists were constructed which had 8 experimental dialogues per condition. No list contained more than one version of a given item. Added to these 48 dialogues were another 32 dialogues from a related experiment (for details, see Hoeks et al. 2007). The order in which experimental and filler items appeared was determined semi-randomly and was the same for each list. Each list was presented to an equal number of participants and each participant saw only one list.

The experimental items for the present experiment appeared in three versions as exemplified below $(3 \mathrm{a}-\mathrm{c})$. Note that English translations are given of the original Dutch stimuli (target sentences are structurally identical between languages) and that CAPITALS indicate the presence of a pitch accent. Probes are given in brackets; first the identical probe, then the control probe: $<$ Wilma/Willem $>$.

\section{3a. (GG) Gapping Context, Gapping Prosody}

Context: Wilma has bought a new house. What did Nathan and Tessa help her with?

Target: NATHAN helped Wilma with PAIN-TING and TESSA with $<$ Wilma/Willem $>$ WALLPAPERING.

Verification Statement (Gapping): Tessa helped Wilma.

3b. (GN) Gapping Context, Nongapping Prosody

Context: Wilma has bought a new house. What did Nathan and Tessa help her with?

Target: Nathan helped WILMA with PAINTING and TESSA with $<$ Wilma/Willem $>$ WALLPAPERING.

Verification Statement (Gapping): Tessa helped Wilma.

3c. (NN) Nongapping Context, Nongapping Prosody

Context: Wilma and Tessa have each bought a new house. What did Nathan help them with?

Target: Nathan helped WILMA with PAINTING and TESSA with $<$ Wilma/Willem $>$ WALLPAPERING.

Verification Statement (Gapping): Tessa helped Wilma.

All verification statements at the end of the dialogues in Experiment 2 represented the gapping reading of the ambiguous target sentence. The verification statements belonging to the fillers, however, all expressed the nongapping reading. As to the probes: control probes were chosen such that they matched the identical probes as closely as possible in terms of number of characters and Google frequency. The practice session that preceded the actual experiment consisted of three parts: In the first part only the gender decision task was practiced, without any auditory input. Then only the auditory decision task with the verification statements was practiced, so without the gender decision task. Finally, the combined tasks were practiced.

\section{Procedure}

The procedure was identical to that used in Experiment 1, except that participants had to do the extra task of deciding whether a visually presented name was either a girl's or a boy's 
Table 1 Mean proportions of gapping (SE in parentheses)

\begin{tabular}{lll}
\hline Condition & Experiment 1 & Experiment 2 \\
\hline Gapping context, Gapping prosody ("GG") & $0.61(.05)$ & $0.62(.05)$ \\
Gapping context, Nongapping prosody ("GN") & $0.37(.05)$ & $0.45(.04)$ \\
Nongapping context, Nongapping prosody ("NN") & $0.09(.03)$ & $0.16(.03)$ \\
\hline
\end{tabular}

name: At the off-set of the second preposition in the ambiguous target sentence (e.g., the 'with' of the phrase 'with wallpapering' in 3a-c), either an identical or a control probe was presented which remained on screen until a gender decision was made. For this task, participants also used the SHIFT keys: right SHIFT for "boy's name" and LEFT shift key for "girl's name". To avoid confusion, the SHIFT keys were labeled, and A5-sized pieces of paper were placed to the right and to the left of the keyboard with "yes - boy's name" and "no - girl's name", respectively.

Results

Analysis

Proportions "YES" to the verification statements were calculated for each participant and each item. In this experiment, a "YES" response is always a choice for the gapping reading; "NO" responses are choices for the nongapping reading. In addition, response times to the probes were calculated, but only for correct responses (i.e., where the chosen gender matched the gender of the probe). Mean proportions gapping response are presented in Table 1 together with the data from Experiment 1, to show that the patterns of results are very similar. The gender decision times are presented in Fig. 3.

For all dependent measures, we performed ANOVA's on participant and item means. The factors Condition (see 2a-c: "GG", "GN", and "NN") and Probe Type (identical vs. control) were treated as within-participants and within-items factors.

\section{Proportion Gapping Responses}

The main effect of Condition was highly significant on both participant- and item-analyses $(F 1(2,60)=58.69, p<.001 ; F 2(2,84)=168.61, p<.001)$. All conditions differed significantly, and the highest proportion of gapping responses, $62 \%$, was found in the "GG" condition, where both context and prosody promoted gapping.

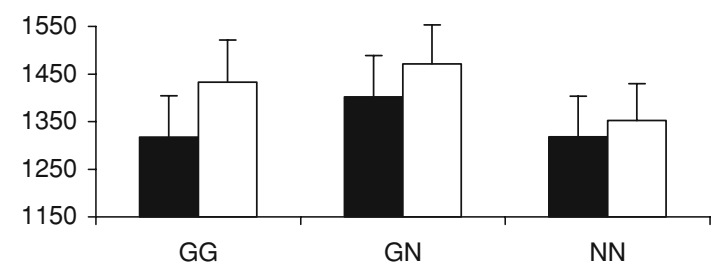

Fig. 3 Decision times for correct responses to the gender probe. "GG" = Gapping context, Gapping prosody; "GN"= Gapping context, Nongapping prosody; "NN"= Nongapping context, Nongapping prosody; black bars $=$ identical probe; white bars $=$ control probe. Error bars represent $1 \mathrm{SE}$ 


\section{Gender Decision Times}

Participants were surprisingly good at the dual task: on average they chose the correct gender $96 \%$ of the time. Statistical analysis of the correct gender decision times produced a significant main effect of Probe Type $(F 1(1,30)=11.02, p<.01 ; F 2(1,42)=4.12, p<.05)$, indicating that the decisions to the identical probes $(1346 \mathrm{~ms} ; \mathrm{SE}=83)$ were faster than to the control probes $(1419 \mathrm{~ms} ; \mathrm{SE}=80)$. The size of the identity priming effect did not differ across conditions: There was no statistically reliable interaction of Condition and Probe Type (both $p$-values $>.25)$. Instead, the main effect of Condition was significant $(F 1(2,60)=6.67, p<$ $.01 ; F 2(2,84)=5.45, p<.01)$, indicating that decision times to probes, whether or not they were identical, were slowest in the "GN" condition $(1437 \mathrm{~ms} ; \mathrm{SE}=83)$, but did not differ between "GG" (1375 ms; SE= 85) and "NN" (1335 ms; SE=79). Exploratory analyses where only "YES" responses were included (where we can be relatively certain that the listener's final representation corresponds to the gapping structure) did not produce a different pattern of results.

\section{Discussion Experiment 2}

First of all, the results of the responses to the verification statements constituted an almost perfect replication of the pattern of the off-line results found in Experiment 1. When gapping context and gapping prosody conspired, participants again chose the gapping interpretation for over $60 \%$ of the time, whereas when context and prosody biased towards nongapping, gapping clearly remained unpreferred, and was chosen only $16 \%$ of the time, with the mismatch condition somewhere in between. Taken together with the very few mistakes that were made in the gender decision task, this result shows that participants were able to handle this dual task paradigm extremely well.

With respect to on-line processing, we found a significant identity priming effect, where identical probes were responded to faster than control probes. However, we did not find any difference in size of the priming-effect between the three conditions. Recall that we had assumed that the concepts associated with the gapped elements (e.g., 'helped Wilma' in the example) would have been activated or reactivated during the processing of the gapping interpretation, which should have made it easier to process the identity probe (e.g., 'Wilma') in the condition with most gapping responses (e.g., "GG"). The fact that this did not happen could mean that there actually was no difference in reactivation between the conditions, either because there was no reactivation to speak of in any of the conditions, or because there was just no difference. In that case, the probes will measure processing load rather than activation: when an utterance is embedded in the right context, and spoken with the right prosody, computing the gapping interpretation is as easy as computing the highly preferred nongapping interpretation. The only processing difficulty is found in the mismatch condition, where context and prosody point to different directions.

\section{General Discussion}

The experiments presented here show that nonstructural factors such as context and prosody have strong and independent effects on both off-line preferences and on-line processing of gapping sentences. In the right combination, context and prosody seemingly eliminate all processing difficulty associated with gapping, and make it the structure of choice. Interestingly, pragmatic context seems to be stronger than prosody in its ability to promote gapping: 
there were more gapping responses in the speeded auditory decision task when context was in favor and prosody was not $(37 \%)$ than when prosody was in favor and context was not $(17 \%)$. So even though prosody helps, the pragmatic information in the context appears to be more important. This may in some sense reflect the tendency of listeners to assume that speakers are rational and cooperative (Grice 1975), and that if a question is posed, a speaker will give a relevant answer. Possible prosodic peculiarities will then to a large part be ignored.

What can our results say about the mechanism of human sentence processing? The results we have presented here are in principle compatible with both major theoretical approaches to language processing: the 'syntax-first' model (Frazier 1987), and the 'constraint-based' model (Tanenhaus and Trueswell 1995). In the syntax-first model, syntactic structure building precedes all other kinds of processing, and in case of ambiguity, the simplest structure is always chosen first. In the case at hand, this can be argued to be the nongapping structure, as the description of gapping structures requires more nodes than that of nongapping (note that under other syntactic formalisms it is less clear whether the structures differ in complexity).

Under such a view, a listener always starts out with a nongapping interpretation, and may need to revise that interpretation but only when strong and relevant non-structural information becomes available. Although the data are, in principle, compatible with a syntax-first processing account, we did not find very convincing evidence for reanalysis effects in the online data from Experiment 2. It is true that probe reaction times were numerically somewhat longer in the condition promoting gapping than in the condition promoting nongapping (1375 vs. $1335 \mathrm{~ms}$ ), but the difference is not significant, and one certainly would have expected a much larger reanalysis-effect because of the proposed strength of the syntactic simplicity factor, and of the subsequent syntactic restructuring that has to take place when the nongapping reading is rejected. Nevertheless, context and prosody in combination may have substantially reduced the amount of effort needed for this kind of reanalysis.

The results that we found are also compatible with models that emphasize the simultaneous interaction of different kinds of constraints (e.g., Tanenhaus and Trueswell 1995). To account for the present data, such constraint-based models must assume that the strong factor going against gapping (which may very well be a syntactic one) is nevertheless easily overpowered by contextual and prosodic information: only when the factors in favor of gapping far outweigh the factors against, can lengthy competition effects between the syntactic alternatives be avoided.

To conclude, the present results do not permit a clear answer to the question which model best describes the data. They do show that pragmatic context is a very important determining factor in language processing, which can even outweigh the effects of the infelicitous prosodic realization of an utterance. And together with prosody, it can make gapping the preferred interpretation of a globally ambiguous sentence.

Acknowledgements This research was funded by grant \# 015.001.103 from the Netherlands Organization for Scientific Research, awarded to Petra Hendriks. We are very grateful to Louisa Zijlstra, Tim Bosselaar, Erik-Jan Smits, and Maartje Schreuder for their help with creating the stimuli and running the experiments.

Open Access This article is distributed under the terms of the Creative Commons Attribution Noncommercial License which permits any noncommercial use, distribution, and reproduction in any medium, provided the original author(s) and source are credited.

\section{References}

Carlson, K. (2001). The effects of parallelism and prosody in the processing of gapping structures. Language and Speech, 44, 1-26. doi:10.1177/00238309010440010101. 
Cutler, A., Dahan, D., \& van Donselaar, W. (1997). Prosody in the comprehension of spoken language: A literature review. Language and Speech, 40(2), 141-201.

Frazier, L. (1987). Sentence processing: A tutorial review. In M. Coltheart (Ed.), Attention \& performance XII (pp. 601-681). Hove, UK: Lawrence Erlbaum.

Grice, H. P. (1975). Logic and conversation. In P. Cole \& J. L. Morgan (Eds.), Syntax and semantics, volume 3: Speech acts (pp. 41-58). New York: Academic Press.

Hoeks, J. C. J., Hendriks, P., \& Redeker, G. (2007). Prosody, context, and thematic fit meet "Gapping": The interaction of multiple constraints in spoken sentence comprehension. In D. S. McNamara \& J. G. Trafton (Eds.), Proceedings of the 29th annual conference of the Cognitive Science Society (pp. 1085-1090), Austin, TX.

Hoeks, J. C. J., Hendriks, P., \& Zijlstra, L. J. (2006). The predominance of non-structural factors in the processing of gapping sentences. In R. Sun (Ed.), Proceedings of the 28th annual conference of the Cognitive Science Society (pp. 1511-1516).

Hoeks, J. C. J., Vonk, W., \& Schriefers, H. (2002). Processing coordinated structures in context. Journal of Memory and Language, 46, 99-119. doi:10.1006/jmla.2001.2800.

Holcomb, P. J. (1993). Semantic priming and stimulus degradation: implications for the role of the N400 in language processing. Psychophysiology, 30(1), 47-61.

Kaan, E., Wijnen, F., \& Swaab, T. Y. (2004). Gapping: Electrophysiological evidence for immediate processing of "missing" verbs in sentence comprehension. Brain and Language, 89, 584-592. doi:10.1016/j.bandl. 2004.02.004.

Keller, F. (2001). Experimental evidence for constraint competition in gapping constructions. In G. Müller \& W. Sternefeld (Eds.), Competition in syntax (pp. 211-248). Berlin: Mouton de Gruyter.

Kuno, S. (1976). Gapping. A functional analysis. Linguistic Inquiry, 7, 300-318.

Lambrecht, K. (1994). Information structure and sentence form. Cambridge, MA: University Press.

Raithel, V., \& Hielscher-Fastabend, M. (2004). Emotional and linguistic perception of prosody. Folia Phoniatrica et Logopaedica, 56, 7-13. doi:10.1159/000075324.

Tanenhaus, M. K., \& Trueswell, J. C. (1995). Sentence comprehension. In J. L. Miller \& P. D. Eimas (Eds.), Speech, language and communication (pp. 217-262). San Diego, CA: Academic Press. 